\title{
OPTION PRICING DRIVEN BY A TELEGRAPH PROCESS WITH RANDOM JUMPS
}

\author{
OSCAR LÓPEZ* AND \\ NIKITA RATANOV, ${ }^{* *}$ Universidad del Rosario
}

\begin{abstract}
In this paper we propose a class of financial market models which are based on telegraph processes with alternating tendencies and jumps. It is assumed that the jumps have random sizes and that they occur when the tendencies are switching. These models are typically incomplete, but the set of equivalent martingale measures can be described in detail. We provide additional suggestions which permit arbitrage-free option prices as well as hedging strategies to be obtained.
\end{abstract}

Keywords: Jump-telegraph process; equivalent martingale measure; option pricing; hedging

2010 Mathematics Subject Classification: Primary 91B28

Secondary 60J27; 60J75

\section{Introduction}

Beginning with the works of Mandelbrot [9] multiple researches have showed that the (geometric) Brownian model for financial data fails to explain the observed behaviour. We propose a construction which solves some difficulties usually attributed to Brownian motion. We use a jump-telegraph process instead of a diffusion process. In the present paper we assume that the jumps are of random sizes. This assumption generalises the (complete) jumptelegraph model with deterministic jumps proposed earlier [15]. As a consequence, the model is incomplete.

Continuous-time telegraph processes have been used before in various probabilistic contexts (see the pioneering works of Goldstein [6] and Kac [8]; see also [12], [13], [18], and [19]).

In the theory of finance, telegraph processes have primarily been used to express stochastic volatility (see [3]). However, a 'telegraph analog' of the Black-Scholes model was considered by, e.g. Di Crescenzo and Pellerey [2] and Pogorui and Rodríguez-Dagnino [14]. It should be noted that the asset pricing models proposed in the above works are based on pure telegraph processes without jump components. It is easy to see that these models have arbitrage opportunities (see [15]).

To avoid arbitrage, a diffusion component can be added to the log-price of a risky asset. For example, Bladt and Padilla [1] considered a (nonarbitrage) model based on a Markov-modulated diffusion process. However, this model is not complete, and so their option pricing formulae depend on undefined switching intensities, $\lambda_{i j}$. Guo [7] proposed a model based on similar principles, but in contrast with [1], added change-of-state security (COS security), i.e. security that pays one unit of account when the Markov chain changes state. This completes the market. For details and comments on this approach, see [16] and the references therein.

Received 14 June 2011; revision received 6 February 2012.

* Postal address: Universidad del Rosario, Calle 12c, No. 4-69, Bogotá, Colombia.

** Email address: nratanov@urosario.edu.co 
Our model is based on a Markov process $\{\varepsilon(t)\}_{t \geq 0}$ with states 0 and 1 , and alternating transition intensities $\lambda_{0}$ and $\lambda_{1}$. We consider the processes $c_{\varepsilon(t)}$ and $r_{\varepsilon(t)}$, and their integrals (integrated telegraph processes)

$$
\mathcal{T}(t)=\int_{0}^{t} c_{\varepsilon(s)} \mathrm{d} s, \quad \mathcal{R}(t)=\int_{0}^{t} r_{\varepsilon(s)} \mathrm{d} s, \quad t \geq 0, r_{0}, r_{1}>0, c_{0}>c_{1} .
$$

To avoid arbitrage opportunities, we exploit the pure jump process in our model. Let

$$
\mathcal{g}(t)=\sum_{1 \leq n \leq N(t)} Y_{\varepsilon\left(\tau_{n}-\right), n},
$$

where $\tau_{n}, n \geq 1$, are the switching times of $\varepsilon(t), N(t)$ is the number of switchings till time $t$, and $Y_{i, n}, n \geq 1, i=0,1$, are independent random variables with alternating distributions supported on $(-1, \infty)$.

We propose the market model defined by a two-dimensional stochastic process $\{B(t)$, $S(t)\}_{t \in[0, T]}$, where $B(t)=\exp (\mathcal{R}(t))$ and $S(t)=S_{0} \mathcal{E}_{t}(\mathcal{T}+\mathcal{g})$. Here $\mathcal{E}_{t}(\cdot)$ is a stochastic exponential.

If the jump values $Y_{i, n}$ are deterministic, the model is complete (see [15]). More precisely, if $Y_{0, n} \equiv y_{0}, Y_{1, n} \equiv y_{1}, y_{0}, y_{1}>-1$, and

$$
\lambda_{0}^{*}:=\frac{r_{0}-c_{0}}{y_{0}}>0, \quad \lambda_{1}^{*}:=\frac{r_{1}-c_{1}}{y_{1}}>0,
$$

then the risk-neutral measure exists and is unique. The switching intensities under the martingale measure are $\lambda_{i}^{*}, i=0,1$. Under conditions (1.1) the option pricing formula was derived in [15]. The option price and hedging strategy of this model depend only on observable parameters, i.e. $r_{i}, c_{i}, y_{i}, i=0,1$, and on the maturity time $T$. These parameters determine the geometry of market movements similar to the technical analysis approach. The important peculiarity of this model is that option prices do not depend on the frequency of jumps. A similar conclusion is obtained by Naik and Lee [11, Section 4] using risk aversion preferences.

The model under consideration does not contain the standard Brownian component, and the log-price is not normally distributed. The simplicity of the model helps to clarify the role of jumps and their pricing. Moreover, our approach allows for diffusion-like behaviour by allowing infinite activity around the origin or, alternatively, by means of normally distributed jump values.

In this paper we present a detailed description of risk-neutral measures in the case of random jump sizes. Moreover, we present a new design of equations for the description of distributions of jump-telegraph processes and option prices. This description is usually based on the PDE technique, but in the present paper we exploit integral equations.

The rest of the paper is organized as follows. In Section 2 we describe the distribution of the telegraph-like process $\mathcal{T}$ with jumps. We also present the necessary and sufficient conditions for the process $\mathcal{T}+\mathcal{g}$ to be a martingale. In Section 3 we first propose an asset pricing model based on the jump-telegraph process and then state our main result, Theorem 3.1, which describes the set of risk-neutral measures for this type of model. In Section 4 we apply Theorem 3.1 to the problem of pricing and hedging European options in the framework of this model. In Section 5 we provide a new version of the fundamental equation. We also construct the self-financing strategies which replicate the European payoff functions when the jump risk is partially insured. These strategies are not typically predictable because of the incompleteness of the model. Concluding remarks and future research are given in Section 6. 


\section{Telegraph processes and martingales}

Let $(\Omega, \mathfrak{F}, \mathrm{P})$ be a probability space. In addition, we fix a time horizon $T$ and a filtration $\left\{\mathfrak{F}_{t}\right\}_{t \in[0, T]}$. Let $\mathfrak{F}=\mathfrak{F}_{T}$.

On the probability space $(\Omega, \mathfrak{F}, \mathrm{P})$ we define the following two independent objects.

- A (right-continuous) Markov process $\varepsilon(t) \in\{0,1\}, t \in[0, T]$, with alternating transition intensities $\lambda_{0}>0$ and $\lambda_{1}>0$ :

$$
\mathrm{P}\{\varepsilon(t+\Delta t) \neq \varepsilon(t) \mid \varepsilon(t)\}=\lambda_{\varepsilon(t)} \Delta t+o(\Delta t) \quad \text { as } \Delta t \rightarrow+0 .
$$

The process $\varepsilon(t), t \in[0, T]$, is assumed to be adapted to the filtration $\left\{\mathfrak{F}_{t}\right\}_{t \in[0, T]}$.

- A sequence of independent random variables $Y_{i, n}$ with alternating distributions $\Phi_{0}(\mathrm{~d} y)$ and $\Phi_{1}(\mathrm{~d} y): \mathrm{P}\left\{Y_{i, n} \in \mathrm{d} y\right\}=\Phi_{i}(\mathrm{~d} y), i=0,1, n \geq 1$. Suppose that $h_{i}:=\mathrm{E} Y_{i, n}=$ $\int_{-\infty}^{\infty} y \Phi_{i}(\mathrm{~d} y)<\infty$.

Note that the switching times $0=\tau_{0}<\tau_{1}<\tau_{2}<\cdots$ of the Markov process $\varepsilon=\varepsilon(t)$ have exponentially distributed increments, i.e. $\mathrm{P}\left\{\tau_{n+1}-\tau_{n}>t \mid \mathfrak{F}_{\tau_{n}}\right\}=\exp \left(-\lambda_{\varepsilon\left(\tau_{n}\right)} t\right)$, and $\tau_{n+1}-\tau_{n}, n \geq 0$, are independent.

Let $\{N(t)\}_{t \in[0, T]}$ be a counting Poisson process defined as

$$
N(t)=\max \left\{n: \tau_{n} \leq t\right\} .
$$

Next, we define a telegraph process $\{\mathcal{T}(t)\}_{t \in[0, T]}$ as

$$
\mathcal{T}(t)=\int_{0}^{t} c_{\varepsilon(s)} \mathrm{d} s
$$

and a compound Poisson process $\{\mathscr{g}(t)\}_{t \in[0, T]}$ as

$$
\mathscr{g}(t)=\sum_{n=1}^{N(t)} Y_{\varepsilon_{n}, n}
$$

Here the constants $c_{0}, c_{1} \in \mathbb{R}$ are the velocity values of the telegraph process, $c_{1}<c_{0}$, and $\varepsilon_{n}=\varepsilon\left(\tau_{n}-\right), n=1,2, \ldots$, is the state of the Markov process $\varepsilon$ just before the $n$th switching.

Processes $\mathcal{T}(t)$ and $\mathscr{g}(t), t \in[0, T]$, are adapted to the filtration $\left\{\mathfrak{F}_{t}\right\}_{t \in[0, T]}$. Note that $\mathfrak{F}_{0}$ is not trivial. Moreover, for any $t>0$, the distributions of $\mathcal{T}(t)$ and $\mathscr{f}(t)$ are completely determined by the initial state $\varepsilon(0) \in\{0,1\}$.

Fix the initial state $\varepsilon(0)=i \in\{0,1\}$. Then, for any $t>0$, we have the equality in distribution

$$
\mathcal{T}(t)+\mathcal{g}(t) \stackrel{\mathrm{D}}{=} c_{i} t \mathbf{1}_{\left\{\tau_{1}>t\right\}}+\left[c_{i} \tau_{1}+Y_{i, 1}+\tilde{\mathcal{T}}\left(t-\tau_{1}\right)+\tilde{\mathscr{g}}\left(t-\tau_{1}\right)\right] \mathbf{1}_{\left\{\tau_{1}<t\right\}},
$$

where the telegraph process $\widetilde{\mathcal{T}}$ and jump process $\tilde{\mathcal{g}}$ are initiated from the opposite state, $1-i$, and they are independent of $\mathcal{T}$ and $\mathcal{g}$. Here $\tau_{1}$ is the first switching time, and $Y_{i, 1}$ is the value of the first jump.

Theorem 2.1. The transition densities $p_{i}(x, t):=\mathrm{P}\{\mathcal{T}(t)+\mathcal{g}(t) \in \mathrm{d} x \mid \varepsilon(0)=i\} / \mathrm{d} x$, $i=0,1$, follow the set of integral equations

$$
p_{i}(x, t)=\mathrm{e}^{-\lambda_{i} t} \delta\left(x-c_{i} t\right)+\int_{0}^{t}\left(\int_{-\infty}^{\infty} p_{1-i}\left(x-c_{i} s-y, t-s\right) \Phi_{i}(\mathrm{~d} y)\right) \lambda_{i} \mathrm{e}^{-\lambda_{i} s} \mathrm{~d} s
$$

for $t \geq 0$ and $i=0,1$, where $\delta(\cdot)$ is Dirac's delta function. 
The expectations $m_{i}(t)=\mathrm{E}\{\mathcal{T}(t)+\mathcal{g}(t) \mid \varepsilon(0)=i\}, t \geq 0, i=0,1$, satisfy

$$
m_{i}(t)=\left(\frac{c_{i}}{\lambda_{i}}+h_{i}\right)\left(1-\mathrm{e}^{-\lambda_{i} t}\right)+\int_{0}^{t} m_{1-i}(t-s) \lambda_{i} \mathrm{e}^{-\lambda_{i} s} \mathrm{~d} s, \quad i=0,1,
$$

where $h_{i}=\mathrm{E}\left\{Y_{i, 1}\right\}=\int_{-\infty}^{\infty} y \Phi_{i}(\mathrm{~d} y), i=0,1$.

Proof. Equality (2.4) follows from (2.3). To compute $m_{i}(t)$, we use (2.4):

$$
\begin{aligned}
m_{i}(t) & =\int_{-\infty}^{\infty} x p_{i}(x, t) \mathrm{d} x \\
& =c_{i} t \mathrm{e}^{-\lambda_{i} t}+\int_{-\infty}^{\infty} x\left[\int_{0}^{t}\left(\int_{-\infty}^{\infty} p_{1-i}\left(x-c_{i} s-y, t-s\right) \Phi_{i}(\mathrm{~d} y)\right) \lambda_{i} \mathrm{e}^{-\lambda_{i} s} \mathrm{~d} s\right] \mathrm{d} x \\
& =c_{i} t \mathrm{e}^{-\lambda_{i} t}+\int_{-\infty}^{\infty}\left[\int_{0}^{t} \lambda_{i} \mathrm{e}^{-\lambda_{i} s}\left(\int_{-\infty}^{\infty} x p_{1-i}\left(x-c_{i} s-y, t-s\right) \mathrm{d} x\right) \mathrm{d} s\right] \Phi_{i}(\mathrm{~d} y) .
\end{aligned}
$$

Applying the change of variable $x=x^{\prime}+c_{i} s+y$ we have

$$
\begin{aligned}
m_{i}(t)= & c_{i} t \mathrm{e}^{-\lambda_{i} t}+c_{i} \int_{0}^{t} s \lambda_{i} \mathrm{e}^{-\lambda_{i} s} \mathrm{~d} s+\int_{-\infty}^{\infty} y \Phi_{i}(\mathrm{~d} y) \int_{0}^{t} \lambda_{i} \mathrm{e}^{-\lambda_{i} s} \mathrm{~d} s \\
& +\int_{0}^{t} m_{1-i}(t-s) \lambda_{i} \mathrm{e}^{-\lambda_{i} s} \mathrm{~d} s \\
= & \left(\frac{c_{i}}{\lambda_{i}}+h_{i}\right)\left(1-\mathrm{e}^{-\lambda_{i} t}\right)+\int_{0}^{t} m_{1-i}(t-s) \lambda_{i} \mathrm{e}^{-\lambda_{i} s} \mathrm{~d} s .
\end{aligned}
$$

Integral equations (2.5) can be rewritten in differential form.

Corollary 2.1. The set of equations (2.5) is equivalent to the Cauchy problem

$$
\frac{\mathrm{d} m_{i}}{\mathrm{~d} t}=c_{i}+\lambda_{i} h_{i}-\lambda_{i} m_{i}(t)+\lambda_{i} m_{1-i}(t), \quad t>0, i=0,1,
$$

with initial conditions $m_{0}(0)=m_{1}(0)=0$.

Proof. The initial conditions follow directly from (2.5). Differentiating (2.5) and then evaluating the integral using integration by parts, with initial conditions $m_{0}(0)=m_{1}(0)=0$, yields

$$
\begin{aligned}
\frac{\mathrm{d} m_{i}(t)}{\mathrm{d} t} & =\left(\frac{c_{i}}{\lambda_{i}}+h_{i}\right) \mathrm{e}^{-\lambda_{i} t}-\int_{0}^{t} \frac{\partial m_{1-i}(t-s)}{\partial s} \lambda_{i} \mathrm{e}^{-\lambda_{i} s} \mathrm{~d} s \\
& =\left(\frac{c_{i}}{\lambda_{i}}+h_{i}\right) \mathrm{e}^{-\lambda_{i} t}+\lambda_{i} m_{1-i}(t)-\lambda_{i} \int_{0}^{t} m_{1-i}(t-s) \lambda_{i} \mathrm{e}^{-\lambda_{i} s} \mathrm{~d} s \\
& =c_{i}+\lambda_{i} h_{i}-\lambda_{i} m_{i}(t)+\lambda_{i} m_{1-i}(t) .
\end{aligned}
$$

Remark 2.1. The exact forms of the distribution densities $p_{i}(x, t)$ (with deterministic jump values) and the expectations $m_{i}(t)$ of the jump-telegraph processes are known; see [15].

The next theorem could be considered as a version of the Doob-Meyer decomposition for jump-telegraph processes with alternating intensities. 
Theorem 2.2. The process $\{\mathcal{T}(t)+\mathcal{G}(t)\}_{t \in[0, T]}$ is a martingale if and only if

$$
c_{0}+\lambda_{0} h_{0}=0 \text { and } c_{1}+\lambda_{1} h_{1}=0 .
$$

Proof. First note that, for $s, t \in[0, T], s<t$,

$$
\mathrm{E}\left\{\mathcal{T}(t)+\mathcal{g}(t) \mid \mathfrak{F}_{s}\right\}=\mathcal{T}(s)+\mathscr{g}(s)+m_{\varepsilon(s)}(t-s) .
$$

Next, the solution of the Cauchy problem for system (2.6) with zero initial conditions is equal to $0, m_{i}(t) \equiv 0, i=0,1$, if and only if the equalities in (2.7) hold.

\section{Market model and risk-neutral measures}

Let $\{\mathcal{T}(t)\}_{t \in[0, T]}$ be the telegraph process, and let $\{\mathscr{g}(t)\}_{t \in[0, T]}$ be the jump process defined on the filtered probability space $\left(\Omega, \mathfrak{F},\left\{\mathfrak{F}_{t}\right\}_{t \in[0, T]}, \mathrm{P}\right.$ ) (see (2.1) and (2.2)). We construct the financial market model with two assets.

Assume that the prices of the risky asset vary with the stochastic exponential of $\mathcal{T}+\mathcal{g}$, i.e.

$$
S(t)=S_{0} \varepsilon_{t}(\mathcal{T}+\mathcal{g})=S_{0} \exp (\mathcal{T}(t)) \prod_{n=1}^{N(t)}\left(1+Y_{\varepsilon_{n}, n}\right)
$$

when the initial price $S(0)=S_{0}>0$ and $\varepsilon_{n}=\varepsilon\left(\tau_{n}-\right)$. The jump values $\left\{Y_{i, n}\right\}_{(i=0,1, n \geq 1)}$ are assumed to be supported on $(-1,+\infty)$.

The bank account $B(t), t \in[0, T]$, yields alternating interest rates $r_{0}, r_{1}>0$, so

$$
B(t)=\exp (\mathcal{R}(t))
$$

where $\mathcal{R}(t)=\int_{0}^{t} r_{\varepsilon(s)} \mathrm{d} s$.

Model (3.1)-(3.2) has no arbitrage possibilities, if the process

$$
B(t)^{-1} S(t)=S_{0} \exp (\mathcal{T}(t)-\mathcal{R}(t)) \prod_{n=1}^{N(t)}\left(1+Y_{\varepsilon_{n}, n}\right)
$$

is martingale with respect to some equivalent measure $\widetilde{\mathrm{P}}$. Here $\mathcal{T}(t)-\mathcal{R}(t), t \in[0, T]$, is the telegraph process with velocities $\bar{c}_{i}:=c_{i}-r_{i}, i=0,1$, which is driven by the Poisson process $N(t), t \in[0, T]$, with alternating intensities $\lambda_{0}, \lambda_{1}>0$.

Theorem 3.1. Model (3.1)-(3.2) possesses an equivalent martingale measure $\widetilde{\mathrm{P}}$ if and only if there exists a pair of positive functions $\varphi_{0}$ and $\varphi_{1}$ such that

$$
\bar{c}_{0}+\int_{-1}^{\infty} y \varphi_{0}(y) \Phi_{0}(\mathrm{~d} y)=0 \text { and } \bar{c}_{1}+\int_{-1}^{\infty} y \varphi_{1}(y) \Phi_{1}(\mathrm{~d} y)=0 .
$$

Under measure $\widetilde{\mathrm{P}}$, the Poisson rates are $\lambda_{i}^{*}=\int_{-1}^{\infty} \varphi_{i}(y) \Phi_{i}(\mathrm{~d} y)$ and the jump values are distributed as $\Phi_{i}^{*}(\mathrm{~d} y)=\left(\varphi_{i}(y) / \lambda_{i}^{*}\right) \Phi_{i}(\mathrm{~d} y), i=0,1$.

Proof. We construct an equivalent probability measure on $\left(\Omega, \mathfrak{F},\left\{\mathfrak{F}_{t}\right\}_{t \in[0, T]}\right)$ in two steps.

Step 1. Consider the constants $c_{i}^{*} \in \mathbb{R}$ and $h_{i}^{*}>-1$ such that $c_{i}^{*}=-\lambda_{i} h_{i}^{*}, i=0,1$. Let $\mathcal{T}^{*}(t)$ be the telegraph process defined as in (2.1) with velocities $c_{0}^{*}$ and $c_{1}^{*}$ instead of $c_{0}$ and $c_{1}$, and let $g^{*}(t)=\sum_{n=1}^{N(t)} h_{\varepsilon_{n}}^{*}$ be the pure jump process with constant jump values $h_{0}^{*}$ and $h_{1}^{*}$. 
Owing to Theorem 2.2, the process $\mathcal{T}^{*}+g^{*}$ is a martingale. Let $Z=Z(t)$ be the stochastic exponential

$$
Z(t):=\varepsilon_{t}\left(\mathcal{T}^{*}+g^{*}\right)=\mathrm{e}^{\mathcal{T}^{*}(t)} \prod_{n=1}^{N(t)}\left(1+h_{\varepsilon_{n}}^{*}\right) .
$$

As the first step, we transform the underlying measure $\mathrm{P}$ into the equivalent probability measure $\mathrm{P}^{*}$ on $\left(\Omega, \mathfrak{F},\left\{\mathfrak{F}_{t}\right\}_{t \in[0, T]}\right)$ by means of the density

$$
\left.\frac{\mathrm{dP}^{*}}{\mathrm{dP}}\right|_{\mathfrak{F}_{t}}=Z(t) .
$$

It is known (see [15, Theorem 3]) that, under the new measure $\mathrm{P}^{*}$, the switching intensities of process $\varepsilon$ become

$$
\lambda_{i}^{*}=\lambda_{i}-c_{i}^{*}=\lambda_{i}\left(1+h_{i}^{*}\right), \quad i=0,1 .
$$

Therefore, we can replace the switching intensities $\lambda_{0}$ and $\lambda_{1}$ by arbitrary $\lambda_{0}^{*}>0$ and $\lambda_{1}^{*}>0$.

Step 2. We pass to the equivalent jump size distributions $\Phi_{i}^{*}(\mathrm{~d} y)$ by virtue of

$$
\Phi_{i}^{*}(\mathrm{~d} y)=\varphi_{i}^{*}(y) \Phi_{i}(\mathrm{~d} y)
$$

with a positive integrable function $\varphi_{i}^{*}(y)$ such that $\int_{-1}^{\infty} \varphi_{i}^{*}(y) \Phi_{i}(\mathrm{~d} y)=1$.

Carrying out these two steps yields the new measure $\widetilde{\mathrm{P}}$ which is equivalent to the original measure P.

Using Theorem 2.2, it is easy to see that the process $\mathcal{T}-\mathcal{R}+\mathcal{g}$ as well as $B^{-1} S$ are $\widetilde{\mathrm{P}}$-martingales if and only if

$$
c_{i}-r_{i}+\lambda_{i}^{*} \int_{-1}^{\infty} y \varphi_{i}^{*}(y) \Phi_{i}(\mathrm{~d} y)=0, \quad i=0,1 .
$$

Hence, model (3.1)-(3.2) possesses an equivalent martingale measure if and only if system (3.3) has an integrable solution $\varphi_{0}(y), \varphi_{1}(y)$. This measure is constructed above: in the first step we create new switching intensities $\lambda_{0}^{*}$ and $\lambda_{1}^{*}$, and in the second step we change the distributions of the jump values to get the density $\varphi_{i}^{*}$ such that $\varphi_{i}^{*}(y)=\varphi_{i}(y) / \lambda_{i}^{*}, i=0,1$.

Integrating the latter equality with respect to the measure $\Phi_{i}(\mathrm{~d} y)$ we obtain

$$
\lambda_{i}^{*}=\int_{-1}^{\infty} \varphi_{i}(y) \Phi_{i}(\mathrm{~d} y), \quad i=0,1,
$$

completing the proof.

Remark 3.1. In the particular case when the jump values are deterministic, i.e. $\Phi_{i}(\mathrm{~d} y)=$ $\delta\left(y-h_{i}\right) \mathrm{d} y$, the equations in (3.3) have the form $\bar{c}_{i}+\lambda_{i}^{*} h_{i}=0, i=0,1$. In this case Theorem 3.1 is equivalent to Theorem 3 of [15].

\section{Pricing European options}

Generally speaking, the set of equations (3.3) may have infinitely many solutions. In order to determine the risk-neutral measure, we should assume an additional condition.

\subsection{Jump risk is not priced}

Following Merton's arguments (see [5] and [10]) we suppose that the telegraph component $\mathcal{T}$ and jump component $g$ respectively represent the systematic risk and unsystematic risk of the 
underlying risky asset. So, we assume that, under the change of measure, the distributions of the jump values are invariant. This assumption is quite reasonable because in the case of the complete market model (when the alternating jump values are deterministic), under the unique martingale measure, only jump intensities are changed [15].

If the distributions of the jump values are not changed, then we consider only constant solutions of the equations in (3.3), i.e. $\varphi_{0}=\lambda_{0}^{*}$ and $\varphi_{1}=\lambda_{1}^{*}$, where $\lambda_{0}^{*}=-\bar{c}_{0} / h_{0}$ and $\lambda_{1}^{*}=-\bar{c}_{1} / h_{1}$ are the new switching intensities, and $h_{i}=\mathrm{E} Y_{i, n}, i=0,1$. The risk neutral measure $\mathrm{P}^{*}$ is defined by (3.4), and, under this measure, the underlying Markov process $\varepsilon$ has the intensities $\lambda_{0}^{*}$ and $\lambda_{1}^{*}$.

The above allows us to write down exact formulae for the option prices. Consider the function $f: \mathbb{R}_{+} \rightarrow \mathbb{R}_{+}$, and the European option with maturity time $T$ and payoff function $X=f(S(T))$. The option can now be priced as the expectation with respect to the risk neutral measure $\mathrm{P}^{*}$, i.e. $c=\mathrm{E}^{*}\left\{\mathrm{e}^{-\mathcal{R}(T)} f(S(T))\right\}$.

\subsection{Jump risk is insured}

To make another risk-neutral measure choice, we supply the market with an additional security which magnifies its value by a fixed rate each time there is a change of state, i.e.

$$
\hat{S}(t)=\prod_{n=1}^{N(t)}\left(1+\hat{h}_{\varepsilon_{n}}\right) .
$$

The process $\{\hat{S}(t)\}_{t \geq 0}$ is governed by the same Poisson process $N$ and it has deterministic jump values $\hat{h}_{0}, \hat{h}_{1}>0$. This security can be considered as an insurance contract which compensates losses and gains provoked by state changes and helps to hedge the option with payoff function $X=f(S(T), \hat{S}(T))$.

A market formed by $\{B(t), S(t), \hat{S}(t)\}, t \in[0, T]$, is still incomplete, but now we can use the following approach to make a reasonable choice of risk-neutral measure. First, we change the measure with respect to the switching intensities. Applying Theorem 3.1 to the asset $\hat{S}$ and using Remark 3.1, we define the equivalent measure with switching intensities $\lambda_{i}^{*}=r_{i} / \hat{h}_{i}$. We then make one more change of measure, conserving the form of the distribution of the jump values $Y_{i, n}, i=0,1, n \in \mathbb{N}$.

We now give some examples illustrating this approach.

Example 4.1. (Exponential distribution.) Assume that $\bar{c}_{0}, \bar{c}_{1}<0$ and that the alternating distributions of the jump sizes (under the measure P) are exponential with densities $\Phi_{i}(\mathrm{~d} y)=$ $\eta_{i} \mathrm{e}^{-\eta_{i} y} \mathbf{1}_{\{y>0\}} \mathrm{d} y, \eta_{i}>0, i=0,1$. Assuming that these distributions, under the martingale measure $\widetilde{\mathrm{P}}$, are also exponential, we set $\varphi_{i}(y)=\alpha_{i} \mathrm{e}^{-a_{i} y} \mathbf{1}_{\{y>0\}}, a_{i}>-\eta_{i}, \alpha_{i}>0$. From the equations in (3.3) we have $a_{i}=-\eta_{i}+\sqrt{-\alpha_{i} \eta_{i} / \bar{c}_{i}}$.

Under the martingale measure $\widetilde{\mathrm{P}}$, the new switching intensities are

$$
\lambda_{i}^{*}=\sqrt{-\bar{c}_{i} \eta_{i} \alpha_{i}}
$$

(see (3.6)), and the jump sizes are distributed according to (3.5). Hence, $\alpha_{i}=\lambda_{i}^{* 2} /\left(-\bar{c}_{i} \eta_{i}\right)$, and the new distributions of the sizes of the jumps are

$$
\Phi_{i}^{*}(\mathrm{~d} y)=\eta_{i}^{*} \mathrm{e}^{-\eta_{i}^{*} y} \mathbf{1}_{\{y>0\}} \mathrm{d} y, \quad i=0,1,
$$

where $\eta_{i}^{*}=-\lambda_{i}^{*} / \bar{c}_{i}$. Here $\lambda_{i}^{*}, i=0,1$, are defined as $\lambda_{i}^{*}=r_{i} / \hat{h}_{i}$. 
Example 4.2. (Log-exponential distribution.) Assume that the distribution of $V_{i, n}=\log (1+$ $\left.Y_{i, n}\right)$ is exponential.

More precisely, if $\bar{c}_{i}<0$ then the distribution of $V_{i, n}$ is exponential. If $\bar{c}_{i}>0$, we assume that the distribution of $-V_{i, n}$ is exponential. For both $\bar{c}_{i}<0$ and $\bar{c}_{i}>0$, we set the density of such a random variable as $\eta_{i} \mathrm{e}^{-\eta_{i} v} \mathbf{1}_{\{v>0\}}, \eta_{i}>0, i=0,1$.

Case 1: $\bar{c}_{i}<0$. Assume that $\eta_{i}>1$. The distribution of jump $Y_{i, n}$ is $\Phi_{i}(\mathrm{~d} y)=$ $\eta_{i}(1+y)^{-\left(1+\eta_{i}\right)} \mathbf{1}_{\{y>0\}} \mathrm{d} y$ and $h_{i}=\mathrm{E} Y_{i, n}=1 /\left(\eta_{i}-1\right)$. Assuming that the distributions of $Y_{i, n}$ under the martingale measure $\widetilde{\mathrm{P}}$ are of the same form, we set $\varphi_{i}(y)=\alpha_{i}(1+y)^{-a_{i}} \mathbf{1}_{\{y>0\}}$, $a_{i}>1-\eta_{i}, \alpha_{i}>0$.

From the equations in (3.3) we obtain $a_{i}=-\eta_{i}+\left(1+\sqrt{\Delta_{i}}\right) / 2$, where $\Delta_{i}=1+4 \alpha_{i} \eta_{i} /\left|\bar{c}_{i}\right|$. Under the martingale measure, the new switching intensities are $\lambda_{i}^{*}=\alpha_{i} \eta_{i} /\left(a_{i}+\eta_{i}\right)=$ $2 \alpha_{i} \eta_{i} /\left(1+\sqrt{\Delta_{i}}\right)$. Hence, $\alpha_{i}=\lambda_{i}^{*}\left(1+\lambda_{i}^{*} /\left|\bar{c}_{i}\right|\right) / \eta_{i}$ and the jump sizes $Y_{i, n}$ are distributed as before, i.e. $\Phi_{i}^{*}(\mathrm{~d} y)=\eta_{i}^{*}(1+y)^{-\left(1+\eta_{i}^{*}\right)} \mathbf{1}_{\{y>0\}} \mathrm{d} y$, where $\eta_{i}^{*}=\alpha_{i} \eta_{i} / \lambda_{i}^{*}=1+\lambda_{i}^{*} /\left|\bar{c}_{i}\right|$ and $\lambda_{i}^{*}=r_{i} / \hat{h}_{i}$.

Case 2: $\bar{c}_{i}>0$ and $\lambda_{i}^{*}=r_{i} / \hat{h}_{i}>\bar{c}_{i}$. Now the distribution of jump $Y_{i, n}$ is $\Phi_{i}(\mathrm{~d} y)=$ $\eta_{i}(1+y)^{\eta_{i}-1} \mathbf{1}_{\{-1<y<0\}} \mathrm{d} y$ and $h_{i}=\mathrm{E} Y_{i, n}=-1 /\left(1+\eta_{i}\right), \eta_{i}>0$. Again, we suppose that, under the martingale measure, the jump sizes are distributed as before. Then $\varphi_{i}(y)=$ $\alpha_{i}(1+y)^{a_{i}} \mathbf{1}_{\{-1<y<0\}}, a_{i}>-\eta_{i}, \alpha_{i}>0$.

Repeating the same calculations as in the $\bar{c}_{i}<0$ case we have $a_{i}=-\eta_{i}+\left(-1+\sqrt{\Delta_{i}}\right) / 2$. The new switching intensities are $\lambda_{i}^{*}=\alpha_{i} \eta_{i} /\left(a_{i}+\eta_{i}\right)=2 \alpha_{i} \eta_{i} /\left(-1+\sqrt{\Delta_{i}}\right)$. Hence, $\alpha_{i}=\lambda_{i}^{*}\left(\lambda_{i}^{*} / \bar{c}_{i}-1\right) / \eta_{i}>0$ and the jump sizes $Y_{i, n}$ are distributed as $\Phi_{i}^{*}(\mathrm{~d} y)=\eta_{i}^{*}(1+$ $y)^{\eta_{i}^{*}-1} \mathbf{1}_{\{-1<y<0\}} \mathrm{d} y$, where $\eta_{i}^{*}=\alpha_{i} \eta_{i} / \lambda_{i}^{*}$ and $\lambda_{i}^{*}=r_{i} / \hat{h}_{i}>\bar{c}_{i}$.

Case 3: $\bar{c}_{i}>0$ and $\lambda_{i}^{*}=r_{i} / \hat{h}_{i}<\bar{c}_{i}$ (the case of arbitrage). In this case the model possesses arbitrage opportunities. If the new distributions of the jump sizes have the same structure then $\tilde{h}_{i}=\widetilde{\mathrm{E}} Y_{i, n}=-1 /\left(1+\eta_{i}^{*}\right)$ and $-1<\tilde{h}_{i}<0$. Hence, in this case we have $\bar{c}_{i}+\lambda_{i}^{*} \tilde{h}_{i}=\bar{c}_{i}-\lambda_{i}^{*} /\left(1+\eta_{i}^{*}\right)>0$ (because $\lambda_{i}^{*}<\bar{c}_{i}$ and $\left.\eta_{I}^{*}>0\right)$. Owing to Theorem 3.1, this means that the model has no risk-neutral measures.

Example 4.3. (Log-normal distribution.) Assume that the distributions of $V_{i, n}=\log \left(1+Y_{i, n}\right)$ are Gaussian, $\mathcal{N}\left(m_{i}, \sigma_{i}^{2}\right)$.

Case 1: $\bar{c}_{i}<\lambda_{i}^{*}=r_{i} / \hat{h}_{i}$. In this case the distribution of jump $Y_{i, n}$ is

$$
\Phi_{i}(\mathrm{~d} y)=\frac{1}{(1+y) \sigma_{i} \sqrt{2 \pi}} \exp \left\{-\frac{\left[\log (1+y)-m_{i}\right]^{2}}{2 \sigma_{i}^{2}}\right\} \mathbf{1}_{\{y>-1\}} \mathrm{d} y,
$$

and $h_{i}=\mathrm{E} Y_{i, n}=\exp \left(m_{i}+\sigma_{i}^{2} / 2\right)-1$.

We change the measure, assuming that the new distributions of $1+Y_{i, n}$ are again log-normal with the same $\sigma_{i}$. To this end, we apply $\varphi_{i}(y)=\alpha_{i} \mathrm{e}^{a_{i} \log (1+y)} \mathbf{1}_{\{y>-1\}}, a_{i} \in \mathbb{R}, \alpha_{i}>0$.

The equations in (3.3) yield

$$
a_{i}=\frac{\log \left(1-\bar{c}_{i} / \lambda_{i}^{*}\right)-m_{i}-\sigma_{i}^{2} / 2}{\sigma_{i}^{2}} .
$$

Hence, the new intensities are

$$
\lambda_{i}^{*}=\alpha_{i} \exp \left(m_{i} a_{i}+\frac{\sigma_{i}^{2} a_{i}^{2}}{2}\right)
$$


and $\alpha_{i}=\lambda_{i}^{*} \exp \left(-m_{i} a_{i}-\sigma_{i}^{2} a_{i}^{2} / 2\right), \lambda_{i}^{*}=r_{i} / \hat{h}_{i}>\bar{c}_{i}$. The jump sizes $Y_{i, n}$ are distributed as

$$
\Phi_{i}^{*}(\mathrm{~d} y)=\frac{1}{(1+y) \sigma_{i} \sqrt{2 \pi}} \exp \left\{-\frac{\left[\log (1+y)-\left(m_{i}+\sigma_{i}^{2} a_{i}\right)\right]^{2}}{2 \sigma_{i}^{2}}\right\} \mathbf{1}_{\{y>-1\}} \mathrm{d} y .
$$

Therefore, $V_{i, n} \sim \mathcal{N}\left(m_{i}+\sigma_{i}^{2} a_{i}, \sigma_{i}^{2}\right)$.

Case 2: $\bar{c}_{i}>\lambda_{i}^{*}=r_{i} / \hat{h}_{i}$ (the case of arbitrage). In this case the model possesses arbitrage opportunities. Similar to the case of arbitrage in Example 4.2, we have $\bar{c}_{i}+\lambda_{i}^{*} \tilde{h}_{i}>0$, where $\tilde{h}_{i}=\widetilde{\mathrm{E}} Y_{i, n}>-1$. Again, by Theorem 3.1, the risk-neutral measure is absent.

Remark 4.1. The option prices in the examples presented above can be computed as expectations with respect to the martingale measure $\widetilde{\mathrm{P}}$, i.e. $c=\widetilde{\mathrm{E}}\left\{\mathrm{e}^{-\mathcal{R}(T)} f(S(T))\right\}$.

\section{Model with insurable jump risk: fundamental equation and hedging strategies}

Consider the market model with insured jump risk formed by the three assets $(B(t), S(t)$, $\hat{S}(t))$ defined on the filtered probability space $\left(\Omega, \mathcal{F},\left\{\mathfrak{F}_{t}\right\}_{t \in[0, T]}, \mathrm{P}\right)$; see (3.1), (3.2), and (4.1).

Let $f: \mathbb{R}_{+}^{2} \rightarrow \mathbb{R}_{+}$be a continuous function. Consider a European option with maturity time $T$ and payoff function $X=f(S(T), \hat{S}(T))$. Let $\widetilde{\mathrm{P}}$ be the equivalent martingale measure in this model.

To price the option, we need to study the master function

$$
F_{i}\left(x_{1}, x_{2}, t\right):=\widetilde{\mathrm{E}}_{i}\left\{\mathrm{e}^{-\mathcal{R}(T-t)} f\left(x_{1} \mathrm{e}^{\mathcal{T}(T-t)} \kappa(T-t), x_{2} \hat{\kappa}(T-t)\right)\right\},
$$

where $\widetilde{\mathrm{E}}_{i}$ denotes the conditional expectation with respect to the risk-neutral measure $\widetilde{\mathrm{P}}$ under the condition $\{\varepsilon(0)=i\}, i=0,1$. Here $\kappa(T-t)=\prod_{n=1}^{N(T-t)}\left(1+Y_{\varepsilon_{n}, n}\right)$ and $\hat{\kappa}(T-t)=$ $\prod_{n=1}^{N(T-t)}\left(1+\hat{h}_{\varepsilon_{n}}\right)$.

Conditioning on the first switching, similar to (2.3), we obtain the system of integral equations

$$
\begin{aligned}
F_{i}\left(x_{1}, x_{2}, t\right)= & \mathrm{e}^{-\left(\lambda_{i}^{*}+r_{i}\right)(T-t)} f\left(x_{1} \mathrm{e}^{c_{i}(T-t)}, x_{2}\right) \\
+ & +\int_{t}^{T} \lambda_{i}^{*} \mathrm{e}^{-\left(\lambda_{i}^{*}+r_{i}\right)(s-t)} \\
& \times\left[\int_{-1}^{\infty} F_{1-i}\left(x_{1}(1+y) \mathrm{e}^{c_{i}(s-t)}, x_{2}\left(1+\hat{h}_{i}\right), s\right) \Phi_{i}^{*}(\mathrm{~d} y)\right] \mathrm{d} s
\end{aligned}
$$

for $t<T$ and $i=0$, 1 , with the terminal conditions $\left.F_{i}\left(x_{1}, x_{2}, t\right)\right|_{t \uparrow T}=f\left(x_{1}, x_{2}\right)$. Here the risk-free intensities $\lambda_{i}^{*}$ are defined by the auxiliary security $\hat{S}, \lambda_{i}^{*}=r_{i} / \hat{h}_{i}, i=0,1$, and the distributions $\Phi_{i}^{*}$ make the process $\left(B(t)^{-1} S(t), B(t)^{-1} \hat{S}(t)\right), t \in[0, T]$, martingale.

This system plays the same role as the fundamental equation in the Black-Scholes model.

Remark 5.1. If the payoff function $f$ is continuously differentiable then (5.1) can be rewritten in an equivalent form, as the system of differential equations (cf. Equation (29) of [17])

$$
\begin{aligned}
& \frac{\partial F_{i}}{\partial t}\left(x_{1}, x_{2}, t\right)+c_{i} x_{1} \frac{\partial F_{i}}{\partial x_{1}}\left(x_{1}, x_{2}, t\right) \\
& \quad=\left(r_{i}+\lambda_{i}^{*}\right) F_{i}\left(x_{1}, x_{2}, t\right)-\lambda_{i}^{*} \int_{-1}^{\infty} F_{1-i}\left(x_{1}(1+y), x_{2}\left(1+\hat{h}_{i}\right), t\right) \Phi_{i}^{*}(\mathrm{~d} y) .
\end{aligned}
$$


Now consider the hedging problem for the option with $\mathfrak{F}_{T}$-measurable payoff function $X$. Let $\Pi_{t}=\left\{\psi_{0}(t), \psi(t), \hat{\psi}(t)\right\}$ be a trading strategy with the strategy value

$$
V_{t}=\psi_{0}(t) B(t)+\psi(t) S(t)+\hat{\psi}(t) \hat{S}(t), \quad t \in[0, T] .
$$

We assume that the process $\Pi_{t}$ is $\mathfrak{F}_{t}$-adapted.

The strategy $\Pi_{t}=\left\{\psi_{0}(t), \psi(t), \hat{\psi}(t)\right\}, t \in[0, T]$, replicates the claim $X$ if its terminal value is equal to the payoff of the option:

$$
V_{T}=X \quad \mathrm{P} \text {-almost surely. }
$$

We assume that $\Pi_{t}, t \in[0, T]$, replicates the claim $X$, and that it is self-financing:

$$
\mathrm{d} V_{t}=\psi_{0}(t) \mathrm{d} B(t)+\psi(t) \mathrm{d} S(t)+\hat{\psi}(t) \mathrm{d} \hat{S}(t), \quad t \in[0, T] .
$$

Since the jump occurring at time $\tau_{n}$ has a nonpredictable value $Y_{i, n}$, we cannot presume that the strategy $\Pi_{t}$ is predictable. We assume that between the switching times $\tau_{n-1}$ and $\tau_{n}$ the portfolio holds $\hat{\psi}\left(\tau_{n-1}\right)$ units of the auxiliary asset, and that the decision is made at time $\tau_{n-1}$. Thus, $\hat{\psi}(t)$ is required to have right-continuous almost-sure paths. Paths of the process $\psi(t)$, $t \in[0, T]$, are assumed to be left-continuous almost surely.

Under these assumptions, (5.4) may be rewritten in integral form:

$$
\begin{aligned}
V_{t}= & V_{0}+\int_{0}^{t} \psi_{0}(s) \mathrm{d} B(s)+\int_{0}^{t} \psi(s) S(s) \mathrm{d} \mathcal{T}(s)+\sum_{n=1}^{N(t)} \psi\left(\tau_{n}\right) Y_{\varepsilon_{n}, n} S\left(\tau_{n}-\right) \\
& +\sum_{n=1}^{N(t)}\left[\hat{\psi}\left(\tau_{n}\right)\left(1+\hat{h}_{\varepsilon_{n}}\right)-\hat{\psi}\left(\tau_{n}-\right)\right] \hat{S}\left(\tau_{n}-\right) .
\end{aligned}
$$

The right-hand term follows from

$$
\begin{aligned}
\int_{0}^{t} \hat{\psi}(s) \mathrm{d} \hat{S}(s) & =\hat{\psi}\left(\tau_{N(t)}\right) \hat{S}\left(\tau_{N(t)}\right)-\hat{\psi}(0) \hat{S}(0) \\
& =\sum_{n=1}^{N(t)} \hat{\psi}\left(\tau_{n}\right) \hat{S}\left(\tau_{n}\right)-\sum_{n=1}^{N(t)} \hat{\psi}\left(\tau_{n}-\right) \hat{S}\left(\tau_{n}-\right) \\
& =\sum_{n=1}^{N(t)}\left[\hat{\psi}\left(\tau_{n}\right)\left(1+\hat{h}_{\varepsilon_{n}}\right)-\hat{\psi}\left(\tau_{n}-\right)\right] \hat{S}\left(\tau_{n}-\right)
\end{aligned}
$$

Using balance equation (5.3), i.e. $\psi_{0}(t)=B(t)^{-1}\left(V_{t}-\psi(t) S(t)-\hat{\psi}(t) \hat{S}(t)\right)$, we rewrite the above equation as

$$
\begin{aligned}
V_{t}= & V_{0}+\int_{0}^{t} r_{\varepsilon(s)} V_{s} \mathrm{~d} s+\int_{0}^{t} \psi(s) S(s)\left(c_{\varepsilon(s)}-r_{\varepsilon(s)}\right) \mathrm{d} s-\int_{0}^{t} \hat{\psi}(s) \hat{S}(s) r_{\varepsilon(s)} \mathrm{d} s \\
& +\sum_{n=1}^{N(t)} \psi\left(\tau_{n}\right) Y_{\varepsilon_{n}, n} S\left(\tau_{n}-\right)+\sum_{n=1}^{N(t)}\left[\hat{\psi}\left(\tau_{n}\right)\left(1+\hat{h}_{\varepsilon_{n}}\right)-\hat{\psi}\left(\tau_{n}-\right)\right] \hat{S}\left(\tau_{n}-\right) .
\end{aligned}
$$

Note that if $\Pi_{t}$ is the hedging strategy for $X$ then the respective strategy value $V_{t}$ can be expressed by means of the functions $F_{i}: V_{t}=F_{\varepsilon(t)}(S(t), \hat{S}(t), t)$; see, e.g. [4, Chapter 7]. 
By Itô's formula we obtain

$$
\begin{aligned}
V_{t}= & V_{0}+\int_{0}^{t} \frac{\partial F_{\varepsilon(s)}}{\partial x_{1}}(S(s), \hat{S}(s), s) S(s) c_{\varepsilon(s)} \mathrm{d} s+\int_{0}^{t} \frac{\partial F_{\varepsilon(s)}}{\partial s}(S(s), \hat{S}(s), s) \mathrm{d} s \\
& +\sum_{n=1}^{N(t)}\left(V_{\tau_{n}}-V_{\tau_{n}-}\right) .
\end{aligned}
$$

Comparing the latter two representations of the strategy value $V_{t}$ with fundamental equation (5.2), between jumps we have

$$
\psi(t) S(t)\left(c_{\varepsilon(t)}-r_{\varepsilon(t)}\right)-\hat{\psi}(t) \hat{S}(t) r_{\varepsilon(t)}=A(t),
$$

where

$$
A(t)=\lambda_{\varepsilon(t)}^{*}\left[F_{\varepsilon(t)}(S(t), \hat{S}(t), t)-\int_{-1}^{\infty} F_{1-\varepsilon(t)}\left(S(t)(1+y), \hat{S}(t)\left(1+\hat{h}_{\varepsilon(t)}\right), t\right) \Phi_{\varepsilon(t)}^{*}(\mathrm{~d} y)\right] .
$$

At jump times $\tau_{n}$,

$$
\psi\left(\tau_{n}\right) Y_{\varepsilon_{n}, n} S\left(\tau_{n}-\right)+\left[\hat{\psi}\left(\tau_{n}\right)\left(1+\hat{h}_{\varepsilon_{n}}\right)-\hat{\psi}\left(\tau_{n}-\right)\right] \hat{S}\left(\tau_{n}-\right)=B_{n},
$$

where $B_{n}=V_{\tau_{n}}-V_{\tau_{n}-}$.

In (5.5) passing to the limit as $t \uparrow \tau_{n}$ yields

$$
\psi\left(\tau_{n}\right) \bar{c}_{\varepsilon_{n}} S\left(\tau_{n}-\right)-\hat{\psi}\left(\tau_{n-1}\right) r_{\varepsilon_{n}} \hat{S}\left(\tau_{n}-\right)=A_{n}, \quad \bar{c}_{\varepsilon_{n}}=c_{\varepsilon_{n}}-r_{\varepsilon_{n}},
$$

where

$$
\begin{aligned}
A_{n}= & \lim _{t \rightarrow \tau_{n}-} A(t) \\
=\lambda_{\varepsilon_{n}}^{*} & {\left[F_{\varepsilon_{n}}\left(S\left(\tau_{n}-\right), \hat{S}\left(\tau_{n}-\right), \tau_{n}-\right)\right.} \\
& \left.\quad-\int_{-1}^{\infty} F_{1-\varepsilon_{n}}\left(S\left(\tau_{n}-\right)(1+y), \hat{S}\left(\tau_{n}-\right)\left(1+\hat{h}_{\varepsilon_{n}}\right), \tau_{n}-\right) \Phi_{\varepsilon_{n}}^{*}(\mathrm{~d} y)\right] .
\end{aligned}
$$

Consequently, from (5.6) and (5.7) we get the portfolio at jump times: for $n \geq 1$,

$$
\begin{aligned}
& \psi\left(\tau_{n}\right)=\left(S\left(\tau_{n}-\right) \bar{c}_{\varepsilon_{n}}\right)^{-1}\left[A_{n}+K_{\varepsilon_{n}} r_{\varepsilon_{n}}\right], \\
& \hat{\psi}\left(\tau_{n}\right)=\hat{S}\left(\tau_{n}\right)^{-1}\left[\left(B_{n}-\frac{A_{n} Y_{\varepsilon_{n}, n}}{\bar{c}_{\varepsilon_{n}}}\right)+K_{\varepsilon_{n}}\left(1-\frac{r_{\varepsilon_{n}} Y_{\varepsilon_{n}, n}}{\bar{c}_{\varepsilon_{n}}}\right)\right] .
\end{aligned}
$$

Here $K_{\varepsilon_{n}}=\hat{\psi}\left(\tau_{n-1}\right) \hat{S}\left(\tau_{n-1}\right), n \geq 2, K_{\varepsilon_{1}}=0$.

The strategy between jumps, $\tau_{n-1}<t<\tau_{n}$ (see (5.5)), is

$$
\psi(t)=\left(S(t) \bar{c}_{\varepsilon_{n}}\right)^{-1}\left[A(t)+K_{\varepsilon_{n}} r_{\varepsilon_{n}}\right], \quad \hat{\psi}(t)=\hat{\psi}\left(\tau_{n-1}\right) .
$$

Remark 5.2. The strategy $\Pi_{t}=\left\{\psi_{0}(t), \psi(t), \hat{\psi}(t)\right\}$ constructed in this section (see (5.8)(5.9)) has a nonpredictable component $\hat{\psi}$ of the security $\hat{S}$ which insured jump risks. To explain it, note that the market enlarged by introducing the financial instrument $\hat{S}$ remains incomplete.

Remark 5.3. If jump sizes are deterministic, i.e. $Y_{i, n}=y_{i}, y_{i} \in(-1, \infty)$, we can presume that the market model is complete and that the strategy is predictable. See [15]. 


\section{Conclusion}

In this paper we proposed a class of financial market models for asset prices that follow an exponential telegraph process with jumps of random amplitudes. This statistical behaviour allowed us to describe a set of risk-neutral measures (Theorem 3.1). We considered two approaches to option pricing that enabled us to obtain the closed formulae: in one approach we used Merton's arguments when the jump risk is not priced and in the other approach we added an additional security which insures jump risks. We also gave a methodology of how to hedge options in the case of insured jump risk.

Other methodologies of how to choose the risk-neutral measure can also be applied, e.g. the minimal entropy martingale measure, but we will discuss these in more detail in future work.

\section{Acknowledgement}

This research was partially supported by Research Fund of Rosario University (FIUR), project DVG097.

\section{References}

[1] Bladt, M. and Padilla, P. (2001). Nonlinear financial models: finite Markov modulation and its limits. In Quantitative Analysis in Financial Markets, Vol. III, ed. M. Avellaneda, World Scientific, River Edge, NJ, pp. 159-171.

[2] Di Crescenzo, A. And Pellerey, F. (2002). On prices' evolutions based on geometric telegrapher's process. Appl. Stoch. Models Business Industry 18, 171-184.

[3] Di Masi, G. B., Kabanov, Y. M. and Runggaldier, W. J. (1994). Mean-variance hedging of options on stocks with Markov volatilities. Theory Prob. Appl. 39, 172-182.

[4] Elliott, R. J. ANd Kopp, P. E. (2005). Mathematics of Financial Markets. Springer, New York.

[5] Elliott, R. J., Siu, T. K., Chan, L. and LaU, J. W. (2007). Pricing options under a generalized Markovmodulated jump-diffusion model. Stoch. Anal. Appl. 25, 821-843.

[6] Goldstein, S. (1951). On diffusion by discontinuous movements, and on the telegraph equation. Quart. J. Mech. Appl. Math. 4, 129-156.

[7] Guo, X. (2001). Information and option pricings. Quant. Finance 1, 38-44.

[8] KaC, M. (1974). A stochastic model related to the telegrapher's equation. Rocky Mountain J. Math. 4, 497-509.

[9] Mandelbrot, B. (1963). The variation of certain speculative prices. J. Business 36, 394-419.

[10] Merton, R. C. (1976). Option pricing when underlying stock returns are discontinuous. J. Financial Econom. 3, 125-144.

[11] NAIK, V. AND LEE, M. (1990). General equilibrium pricing of options on the market portfolio with discontinuous returns. Rev. Financial Studies 3, 493-521.

[12] Orsingher, E. (1990). Probability law, flow function, maximum distribution of wave-governed random motions and their connections with Kirchoff's laws. Stoch. Process. Appl. 34, 49-66.

[13] PInsky, M. A. (1991). Lectures on Random Evolution. World Scientific, River Edge, NJ.

[14] Pogorui, A. A. And Rodríguez-Dagnino, R. M. (2009). Evolution process as an alternative to diffusion process and Black-Scholes formula. Random Operators Stoch. Equat. 17, 61-68.

[15] Ratanov, N. (2007). A jump telegraph model for option pricing. Quant. Finance 7, 575-583.

[16] Ratanov, N. (2010). Option pricing model based on a Markov-modulated diffusion with jumps. Braz. J. Prob. Statist. 24, 413-431.

[17] Ratanov, N. and Melnikov, A. (2008). On financial markets based on telegraph processes. Stochastics 80, 247-268.

[18] WeIss, G. H. (2002). Some applications of persistent random walks and the telegrapher's equation. Physica A 311, 381-410.

[19] ZACKS, S. (2004). Generalized integrated telegraph processes and the distribution of related stopping times. J. Appl. Prob. 41, 497-507. 